\title{
The crystalline structures of the odd alkanes pentane, heptane, nonane, undecane, tridecane and pentadecane monolayers adsorbed on graphite at submonolayer coverages and from the liquid $\dagger$
}

\author{
Thomas Arnold, ${ }^{a}$ Chu Chuan Dong, ${ }^{a}$ Robert K. Thomas, ${ }^{a}$ Miguel A. Castro, ${ }^{b}$ Ana Perdigon, \\ Stuart M. Clarke ${ }^{* c}$ and Akira Inaba ${ }^{d}$ \\ a Physical and Theoretical Chemistry Laboratory, South Parks Road, Oxford, UK \\ ${ }^{b}$ Instituto de Ciencia de Materiales de Sevilla, Avda. Americo Vespucio, Sevilla, Spain \\ ${ }^{c}$ Department of Chemistry and BP Institute, Madingley Rise, Madingley Road, Cambridge, \\ $U K C B 3$ OEZ \\ ${ }^{d}$ Department of Chemistry, Graduate School of Science, Osaka University, Toyonaka, \\ Osaka 560, Japan
}

Received 25th February 2002, Accepted 19th April 2002

First published as an Advance Article on the web 11th June 2002

\begin{abstract}
A combination of neutron and X-ray diffraction has been used to structurally characterise the crystalline monolayer structures of all the alkanes with odd number of carbon atoms in their alkyl chains from pentane to pentadecane adsorbed on graphite. The structures of all the molecules investigated at submonolayer coverages are isomorphous with centred rectangular unit cells containing two molecules per cell in a parallel arrangement. This is a completely different structure from the 'herringbone' arrangement of molecules found for the shorter 'even' alkanes, such as hexane, octane and decane. The monolayers at sub-monolayer coverages are interpreted as uniaxial commensurate with the underlying graphite while those monolayers coexisting with the liquid, while structurally similar, are fully commensurate. The difference between the two structures is a uniaxial compression in the $b$-direction with the monolayers coexisting with the liquids significantly more dense than at submonolayer coverages. In the low coverage structures the 'odd' molecules have an all trans conformation with their extended axes parallel to the surface with the plane of the carbon skeleton also parallel to the graphite surface. At high coverages the carbon skeleton is no longer parallel to the graphite surface but significantly tilted. The longest alkanes, tridecane and pentadecane also show evidence of positional and/or rotational disorder at high coverages.
\end{abstract}

\section{Introduction}

The physisorption of simple molecules, such as alkanes, carboxylic acids and alcohols, from the liquid or binary solutions to solid surfaces is of continuing academic and industrial interest. The structure, composition and behaviour of such layers is often completely different to the bulk behaviour and is the result of the delicate balance of adsorbate and surface interactions which often result in a complex phase behaviour on the surface. In particular, many systems are found to adsorb solid, crystalline monolayers at temperatures where the bulk phases are liquids or solutions. In this work we characterise such solid monolayers formed by the homogolous series of simple nalkanes with odd numbers of carbon atoms in their alkyl chains formed at sub-monolayer and multilayer coverages. This is an important series as the surface-adsorbate interactions are entirely of the van der Waals type and should be understood before addressing chemical species with more complex interactions. In a recent paper we have presented the structures of some shorter even alkanes, hexane, octane, decane, dodecane and tetradecane. ${ }^{1}$ In this work we present a combined X-ray and neutron study of the monolayer structures of a number of the shorter 'odd' alkanes to complete

$\dagger$ Electronic supplementary information (ESI) available: Fractional coordinates of single repeat units of some alkanes at sub-monolayer coverage and of the monolayer coexisting with the liquid. See http://www.suppdata/cp/b2/b201988b/ the study. These diffraction techniques are able to provide detailed crystallographic information about the solid monolayers. Because X-rays are more strongly scattered by heavier elements it is usually preferable to combine X-ray and neutron data in investigations of hydrogen containing species, as we do here. In several cases the monolayer structure deduced from $\mathrm{X}$-rays only has been found to be erroneous as the symmetry of the hydrogen containing parts of a structure had a lower symmetry than that of the heavy elements. ${ }^{2}$

In this work we present detailed structural analysis on the solid monolayers formed by the 'odd' membered alkanes from pentane (C5), to pentadecane (C15) at both submonolayer and multilayer coverages using a combination of neutron diffraction and X-ray diffraction data.

\section{Experimental}

Only a brief outline of the diffraction techniques is presented here (see ref. 3 and 4 for a full description). The apparatus and procedures for such experiments have been described elsewhere. ${ }^{5}$ The instruments used for the neutron diffraction were D20 and D1B at the Institut Laue-Langevin, Grenoble ${ }^{5}$ with incident wavelengths of $0.24 \mathrm{~nm}$ and $0.25 \mathrm{~nm}$ respectively. The X-ray experiments were performed at the Department of Chemistry, Osaka University. This equipment is of the symmetrical transmission type with $\mathrm{Cu} \mathrm{K} \alpha$ radiation, as described previously. ${ }^{3,4}$ Scattering from crystalline two-dimensional 
adsorbed layers gives rise to diffraction peaks ${ }^{6,7}$ with a characteristic saw-tooth line shape. The analysis of such patterns can be used to determine the structure of the layer in a manner analogous to diffraction from three-dimensional crystals. The diffraction pattern from an adsorbed monolayer alone is obtained by subtraction of the scattering from the substrate alone from that of the substrate and adsorbed material together. At higher coverages where a solid monolayer coexists with bulk fluid phase the very broad peak characteristic of the fluid phase can also be removed in a straightforward fashion as described previously. ${ }^{8}$ For X-ray measurements protonated materials were used. For the neutron experiments deuterated alkanes were used to minimise the incoherent scattering background that would have arisen from protonated samples.

The adsorbent used was recompressed exfoliated recompressed graphite Papyex (Le Carbone Lorraine). Several graphite samples were used, each characterised by adsorption of nitrogen, and found to have specific surface areas of 14-31.6 $\mathrm{m}^{2} \mathrm{~g}^{-1}$. The deuterated alkanes were obtained from Cambridge Isotope Laboratories with quoted deuteration levels of $>99 \%$. The graphite substrates were outgassed under vacuum in an oven at $350{ }^{\circ} \mathrm{C}$ before known quantities of alkane were added as liquid by micro-syringe under an inert atmosphere of helium. When dosing the graphite it is convienient to know the approximate number of equivalent monolayers adsorbed. This can be estimated from the areas per molecule, taken from the work of Groszek, ${ }^{9}$ and the specific surface area of the graphite. To optimise the scattering contribution from the adsorbed layer, the quantity of bulk adsorbate was kept to a minimum.

\section{Results}

\section{Low coverage structures}

Fig. 1 presents the experimental X-ray scattering patterns from 0.8 monolayers of (a) pentane, (b) heptane, (c) nonane, (d) undecane, (e) tridecane and (f) pentadecane adsorbed on graphite. Fig. 2 presents the experimental neutron scattering patterns from 0.8 monolayers of (a) pentane, (b) heptane, (c) nonane (d) undecane and (e) tridecane adsorbed on graphite. The X-ray data were collected at $10 \mathrm{~K}$ and the neutron data at a temperature of $50 \mathrm{~K}$. The graphite background has been subtracted in preparing these figures. To include both data from D1B, D20 and X-rays on a similar, reduced scale the $x$-axis is given by, $s=\pi \sin (\theta) / \lambda$, where $\theta$ is half the scattering angle and $\lambda$ the radiation wavelength. The features at approximately $s=0.45 \mathrm{~A}^{-1}$ in the neutron and X-ray patterns respectively arise due to imperfect subtraction of the background. In addition detector instability corrections have been made for the D20 data. The peaks in these figures are then the diffraction from the adsorbed monolayers and have the characteristic asymmetric line shape expected from two-dimensional layers. ${ }^{6,7}$
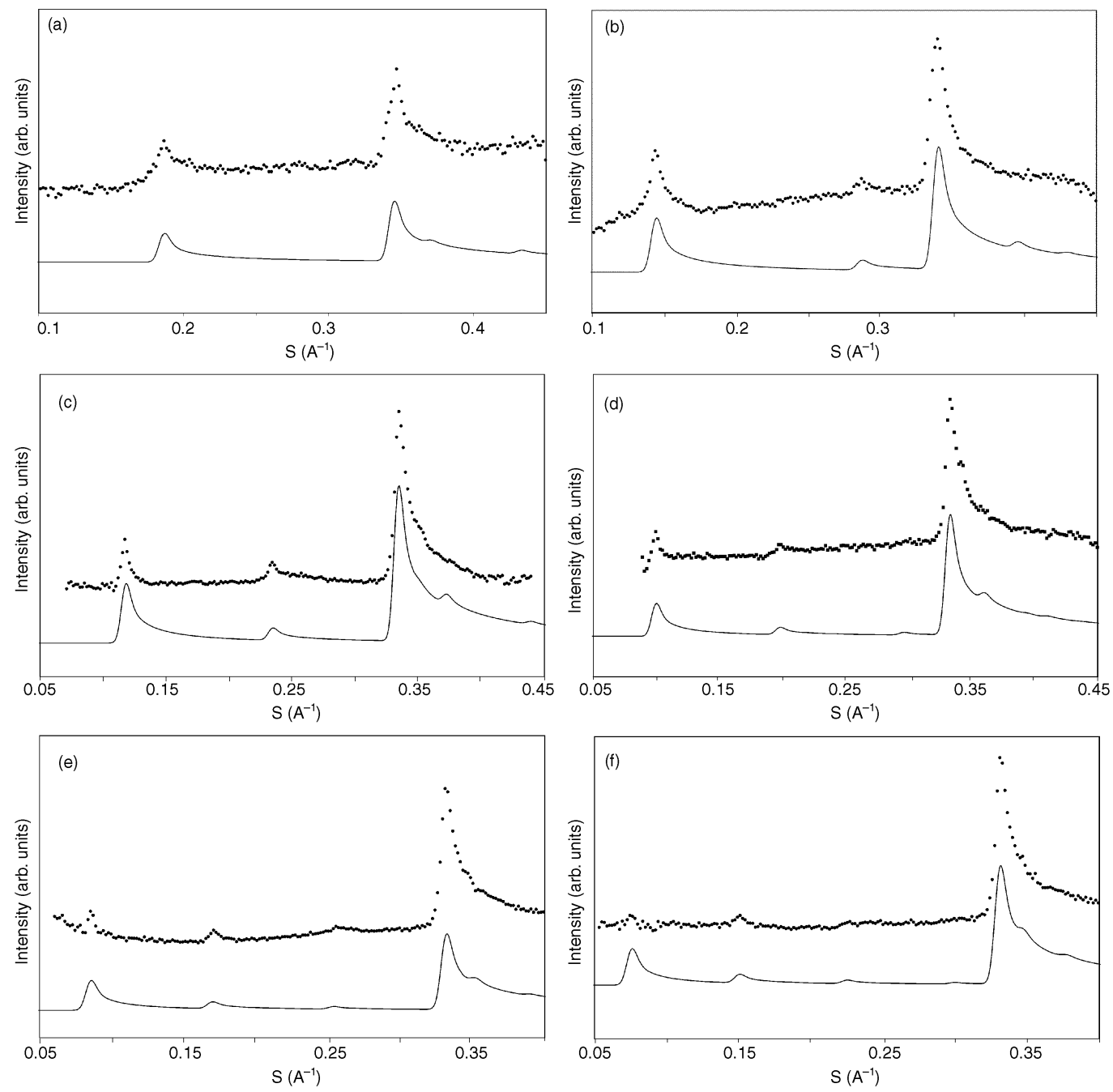

Fig. 1 Experimental (points) and calculated (lines) X-ray diffraction patterns from 0.8 monolayers of (a) pentane, (b) heptane, (c) nonane, (d) undecane (e) tridecane and (f) pentadecane adsorbed on graphite at a temperature of $10 \mathrm{~K}$. The features at approximately $s=0.45 \mathrm{~A}^{-1}$ are regions of imperfect graphite subtraction. 

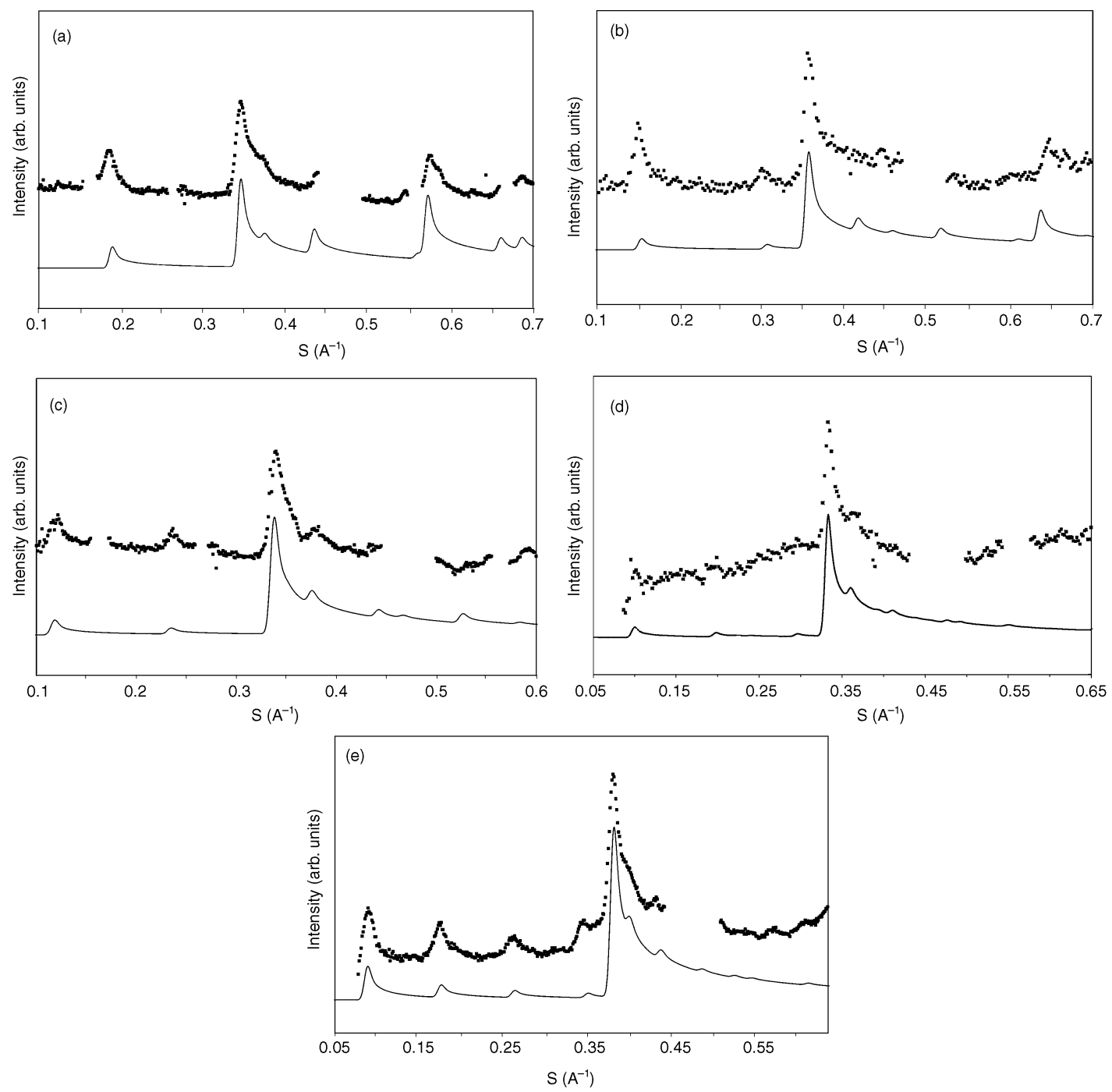

Fig. 2 Experimental (points) and calculated (lines) neutron diffraction patterns from 0.8 monolayers of (a) pentane, (b) heptane, (c) nonane, (d) undecane and (e) tridecane on graphite at a temperature of $50 \mathrm{~K}$. The scattering from the graphite substrate in the absence of any adsorbate has been subtracted in preparing this figure.

Calorimetry data presented previously ${ }^{10}$ has reported that the monolayers of the odd alkanes have an additional phase change close to the monolayer melting transition. Unfortunately we did not have sufficient temperature precision to reliably resolve patterns from these phases in these diffraction experiments.

\section{Coexisting liquids and solid monolayers}

Fig. 3 presents the experimental neutron scattering patterns from solid monolayers of (a) pentane, (b) heptane, (c) nonane, (d) undecane, and (e) tridecane adsorbed on graphite. The temperatures and coverages are: (a) 3 layers at $130 \mathrm{~K}$ (C5), (b) 3 layers at $190 \mathrm{~K}(\mathrm{C} 7)$, (c) 2 layers at $230 \mathrm{~K}$ (C9), (d) 5 layers at $250 \mathrm{~K}(\mathrm{C} 11)$, (e) 3 layers at $285 \mathrm{~K}(\mathrm{C} 13)$. For all these alkanes, except pentane, the monolayer melting temperatures are above the bulk melting point of the alkanes (207 K (C7), $251 \mathrm{~K}(\mathrm{C} 9), 280 \mathrm{~K}(\mathrm{C} 11)$ and $303 \mathrm{~K}(\mathrm{C} 13))$. Hence these solid monolayers coexist with their bulk fluids. As reported previously the monolayer melting point of pentane is found to be the same as the bulk $(188 \mathrm{~K}) .{ }^{4}$ In preparing these figures both the graphite background and the bulk fluid scattering have been subtracted as described previously. ${ }^{11}$ Similar features arising from imperfect subtraction to those present in Fig. 2 are also present in this figure. The peaks in these figures are then the diffraction from the solid adsorbed monolayers that coexist with their liquids.

\section{Structural analysis}

Unlike conventional 3D crystallography, there are usually a much smaller number of reflections in the experimentally determined diffraction patterns from adsorbed monolayers. More independent information is obtained by combining Xray and neutron diffraction data but even then it is not appropriate to fit a large number of structural parameters. In this work we take the conventional approach and attempt to fit the most highly constrained problem first before including additional variables, if required. In this work when fitting the experimental diffraction patterns the molecular bond lengths and angles have been taken to be those of the bulk alkanes $^{12}$ and the alkanes were taken to be adsorbed in the all trans configuration. Refinement of the molecular bond lengths and angles was only made in the final stages of the structural analysis. The diffraction patterns for each trial structure were calculated as described previously ${ }^{13,4}$

\section{Sub-monolayer coverage structures}

Indexing of the experimental reflections and close packing considerations can be used to determine the plane group of the 

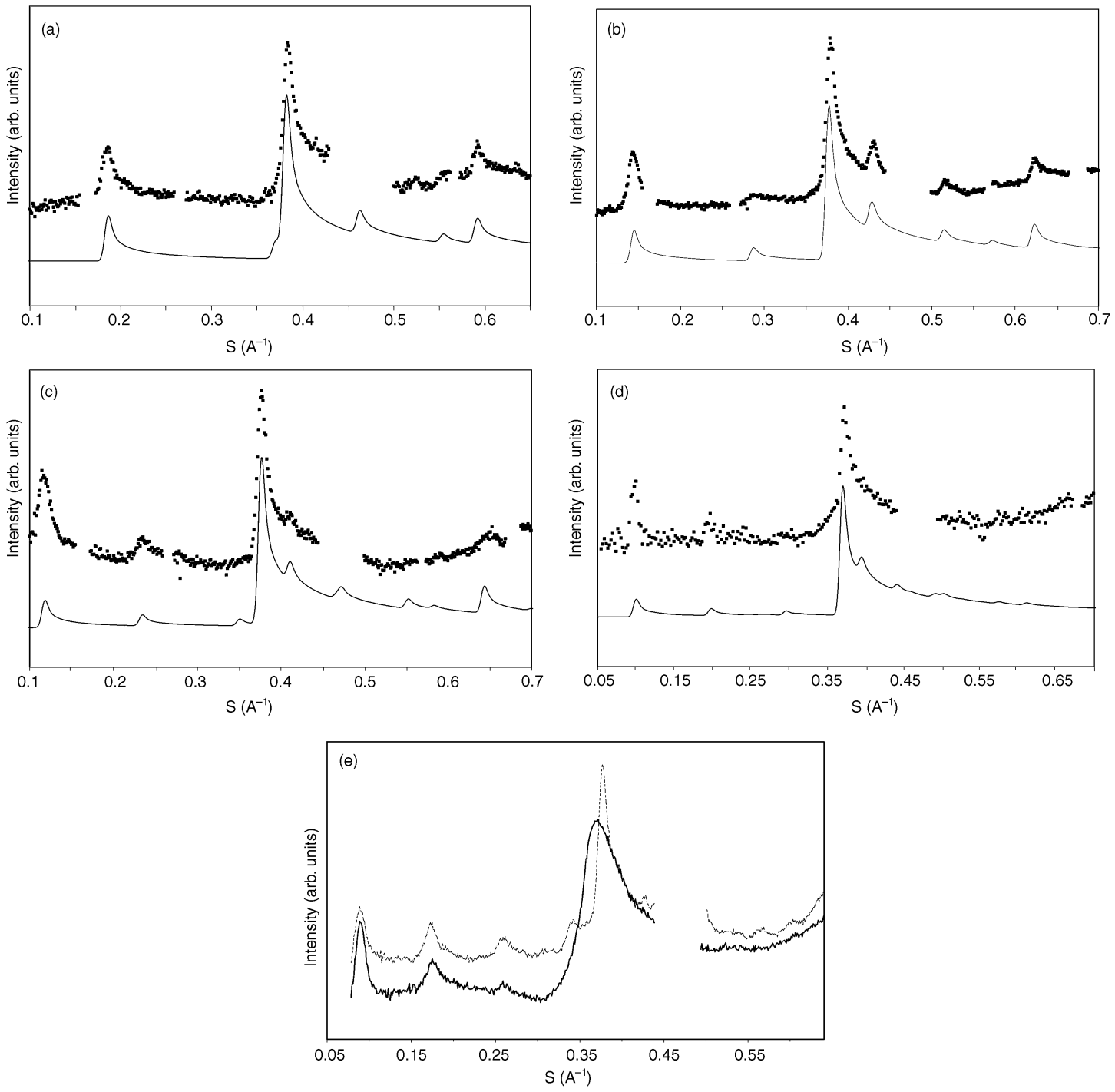

Fig. 3 Experimental (points) and calculated (lines) neutron diffraction patterns from solid monolayers of (a) pentane, (b) heptane, (c) nonane, (d) undecane and (e) tridecane adsorbed on graphite at high coverage. The temperatures and coverages are discussed in the text.

adsorbed layers. ${ }^{4}$ All the unit cells can be indexed on the rectangular unit cells given in Table 1 . We notice that the unit cell $a$-parameters are approximately twice the length of the extended alkane chain indicating that the long axes of the adsorbed alkanes are parallel to the $a$-axes of the unit cell. The systematic absences (reflections $(H, K) H+K=$ odd are absent) indicate that these lattices are centred and so the plane groups of the type pmg can be excluded. If the molecules are initially taken to have their carbon backbone parallel to the graphite surface the plane group must be $\mathrm{cm}$ when the long axis of the molecules are also parallel. The orientation of the carbon backbone on the surface will be discussed further below. The symmetry related positions under $\mathrm{cm}$ are given in Table 2. With this space group and the assumption of a fixed molecular conformation, there are no free parameters and the intensities of the entire set of Bragg peaks for each alkane at this coverage can be calculated. If all the odd alkanes are isomorphic then the relative intensities of all the reflections in all the patterns are now fixed. Remarkably, a good fit to the experimentally determined peak positions and intensities of all the low coverage patterns in both Figs. 1 and 2 were obtained with these highly constrained, isomorphic structures.

Table 1 Summary of the monolayer structures of the odd alkanes pentane, heptane, nonane, undecane, tridecane and pentadecane. Dimensions of the fully commensurate lattices are given in brackets

\begin{tabular}{|c|c|c|c|c|c|}
\hline Alkane & $\begin{array}{l}\text { Low coverage } \\
\text { (X-ray) }\end{array}$ & $\begin{array}{l}\text { Low coverage } \\
\text { (neutron) }\end{array}$ & $\begin{array}{l}\text { Axial } \\
\text { rotation }\end{array}$ & $\begin{array}{l}\text { High coverage } \\
\text { (neutron) }\end{array}$ & $\begin{array}{l}\text { Axial } \\
\text { rotation }\end{array}$ \\
\hline Pentane & $1.71 \mathrm{~nm}$ by $0.475 \mathrm{~nm}$ & $1.67 \mathrm{~nm}$ by $0.47 \mathrm{~nm}$ & 0 & $1.71 \mathrm{~nm}$ by $0.426 \mathrm{~nm} 7$ by $\sqrt{ } 3(1.72 \mathrm{~nm}$ by $0.426 \mathrm{~nm})$ & 30 \\
\hline Heptane & $2.20 \mathrm{~nm}$ by $0.475 \mathrm{~nm}$ & $2.16 \mathrm{~nm}$ by $0.47 \mathrm{~nm}$ & 0 & $2.20 \mathrm{~nm}$ by $0.426 \mathrm{~nm} 9$ by $\sqrt{ } 3(2.2 \mathrm{~nm}$ by $0.426 \mathrm{~nm})$ & 45 \\
\hline Nonane & $2.70 \mathrm{~nm}$ by $0.48 \mathrm{~nm}$ & $2.70 \mathrm{~nm}$ by $0.475 \mathrm{~nm}$ & 0 & $2.70 \mathrm{~nm}$ by $0.424 \mathrm{~nm} 11$ by $\sqrt{ } 3(2.71 \mathrm{~nm}$ by $0.426 \mathrm{~nm})$ & 45 \\
\hline Undecane & $3.20 \mathrm{~nm}$ by $0.48 \mathrm{~nm}$ & $3.20 \mathrm{~nm}$ by $0.48 \mathrm{~nm}$ & 0 & $3.20 \mathrm{~nm}$ by $0.430 \mathrm{~nm} 13$ by $\sqrt{ } 3(3.20 \mathrm{~nm}$ by $0.426 \mathrm{~nm})$ & Disorder \\
\hline Tridecane & $3.74 \mathrm{~nm}$ by $0.48 \mathrm{~nm}$ & $\begin{array}{l}3.80 \mathrm{~nm} \text { by } 0.44 \mathrm{~nm} \\
\quad(\text { see text) }\end{array}$ & 0 & $3.80 \mathrm{~nm}$ by $?$ (see text) & Disorder \\
\hline Penta-decane & $4.22 \mathrm{~nm}$ by $0.48 \mathrm{~nm}$ & & 0 & - & - \\
\hline
\end{tabular}


Table 2 Symmetry operations of the $\mathrm{cm}$ space group

\begin{tabular}{ll}
\hline$x, y$ & $-x,-y$ \\
\hline$\frac{1}{2}+x, \frac{1}{2}+y$ & $\frac{1}{2}-x, \frac{1}{2}-y$ \\
\hline
\end{tabular}

The solid lines in Figs. 1 and 2 represent the calculated profiles and examples of the structures (heptane and nonane) are schematically illustrated in Fig. 4. A final stage of molecular structure refinement has been made in completing this fitting procedure, although the required adjustments to the molecular parameters were small. The fractional coordinates of the repeat motif in the unit cells are given in Tables S1-6.†

The structures obtained by this analysis are physically reasonable with the deuterium atoms of one molecule fitting in the hollows of the adjacent chain. It is interesting that the $a$ axis parameters of the unit cells of all the monolayers are close to integer multiples of $a_{\mathrm{g}}$, where $a_{\mathrm{g}}$ is lattice parameter of the graphite $(0.246 \mathrm{~nm})$. This suggests that the monolayers are uniaxially commensurate with the underlying graphite at low coverages. The molecular orientation with respect to the $a$-axis is also consistent with a commensurate layer as schematically illustrated in Fig. 4.

\section{Monolayers coexisting with liquid}

The reflections in the experimental neutron patterns of Fig. 3 at high coverage can also be assigned on rectangular unit cells given in Table 1. These structures are found to be isomorphic to those at low coverage except that the cells are uniaxially compressed in the $b$-direction and the unit cells are now fully commensurate in both $a$ and $b$ directions. A similar compression from a uniaxial commensurate to fully commensurate lattice is also observed for the shorter even alkanes. ${ }^{1}$ The $b$ parameter appears to be very sensitive to coverage at submonolayer coverages for all the odd alkanes studied here. Indeed, samples of the same alkane at the same nominal coverage have slightly different $b$-values due to small uncertainities in preparing the samples. In particular, the very short $b$-parameter for the neutron pattern of tridecane $(0.44 \mathrm{~nm})$ at a notionally sub-monolayer coverage leads us to suspect that the actual coverage is in fact somewhat higher than 0.8 . The shortest $b$-parameter is found in the commensurate layers at high coverage $(0.426 \mathrm{~nm})$. The longest $b$-parameter found at the lowest coverages investigated here is $0.480 \mathrm{~nm}$. However, this may not represent the longest $b$-axis possible at even lower coverages.

The scattering pattern from tridecane at multilayer coverages show sharp and distinct $(H, 0)$ reflections but reflections of the type $(H, K)$ are rather broad. Fig. 3(e) compares the low and high coverage experimental patterns for tridecane. This indicates that although ordered along the direction parallel

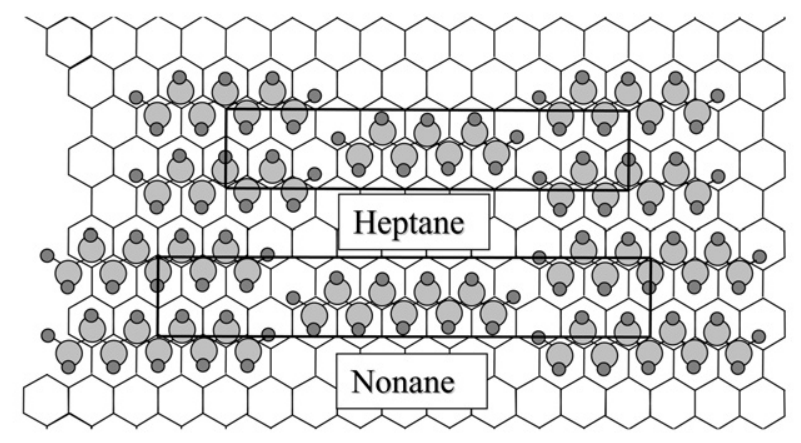

Fig. 4 Schematic representations of the crystal structures deduced for heptane and nonane adsorbed on graphite. These structures are discussed in the text. to the $a$-axis there is significant disorder in the lattice in the $b$-direction. Similar disorder was observed in adsorbed monolayers of the longer even alkanes dodecane and tetradecane. ${ }^{1}$ The disorder and broadening of these reflections means we are unable to determine the $b$-parameter of this unit cell or the molecular orientation within the cell with any confidence. The fractional coordinates of the repeating motifs are given in the ESI Tables S7-10.†

\section{Discussion and conclusions}

The low coverage monolayer structures of the odd alkanes pentane to pentadecane are found to be isomorphous, differing only in by an integral value of $0.492 \mathrm{~nm}$ in the $a$-axis for each additional $\mathrm{CH}_{2} \mathrm{CH}_{2}$. On increasing the coverage above a monolayer there is a uniaxial compression in the $b$-direction.

Sensitivity calculations indicate that the angle the molecules make with respect to the $a$-axis of the unit cell is $0^{\circ} \pm 2$. The orientation of the molecules about their long axes is of some interest. Fig. 5 illustrates the sensitivity of the neutron patterns to rotations about this long molecular axis for pentane. The variations in the intensities of the X-ray patterns are somewhat smaller. In this figure, an axial rotation of zero corresponds to the carbon backbone parallel to the graphite surface and at an axial rotation of 90 the carbon backbones are normal to the surface. Additional calculations also indicate that rotational disorder in the layers cannot be distinguished from the patterns when the carbon backbones are normal to the graphite surface.

For the low coverage structures we conclude that the molecules in the monolayers of pentane, heptane, nonane, tridecane and pentadecane are flat, as illustrated in Fig. 4, with an uncertainty of 20 degrees. The results for undecane were less conclusive with the distinction between patterns calculated for flat and upright molecules being of the same order as the experimental error. However, the intensities are certainly consistent with the flat orientation observed for all the other odd alkanes. For the high coverage monolayers we conclude that the molecules of pentane, heptane and nonane become significantly inclined with respect to the surface, as illustrated in Table 1. Additional independent intensity information was obtained by further neutron measurements made using partially deuterated alkanes, where the relative intensities are expected to be different from the fully deuterated samples for the different axial rotations (e.g. partially deuterated nonane $\mathrm{CH}_{3} \mathrm{CD}_{2} \mathrm{CH}_{2} \mathrm{CD}_{2} \mathrm{CH}_{2} \mathrm{CD}_{2} \mathrm{CH}_{2} \mathrm{CD}_{2} \mathrm{CH}_{3}$ ). However, even with this additional information the molecular orientation about the long molecular axis cannot be determined within less than approximately 20 degrees. Such a reorientation from flat to tilted on increasing the coverage may be necessary to

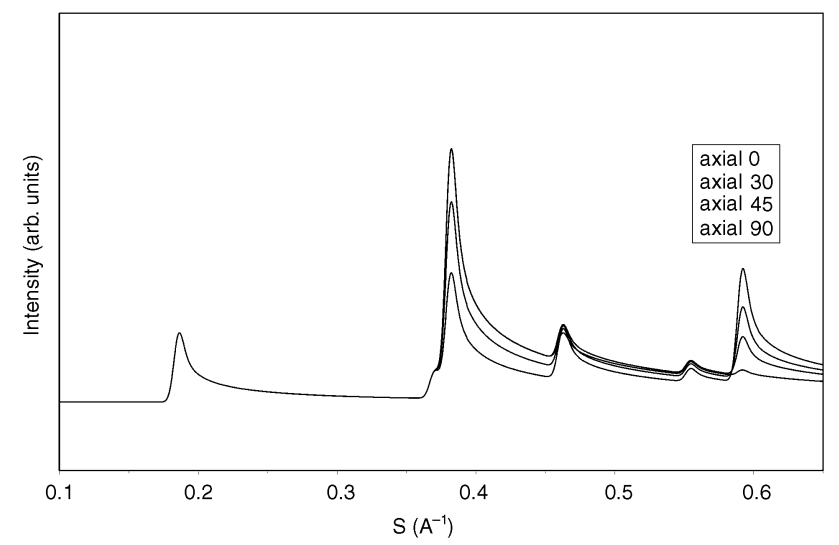

Fig. 5 Figure illustrating the sensitivity of the neutron scattering patterns to the axial rotation of the molecules about their long axes. 
accommodate the compression in the $b$-direction of the lattice on increasing the coverage. Similar tilted arrangements of alkanes are also seen in bulk crystals of alkanes. ${ }^{14}$

A recent STM study ${ }^{15}$ of the C27 alkane has identified lamellar structures with extended carbon chains oriented parallel to the graphite basal plane in good agreement with our results. In the STM study the alkane's chains are almost perpendicular to the lamellae, in good agreement with our diffraction results.

Most significantly the packing of all the odd alkanes presented here are completely different from those of the even alkanes presented previously. ${ }^{1}$ Even alkanes have a 'herringbone' zig-zag (pgg) arrangement of molecules contrasting with the parallel arrangement of the odd alkanes demonstrated here. An exception is the behaviour of the dodecane monolayer which shows two phases. The low temperature phase (II) has a structure isomorphic to the other even alkanes. However, the high temperature phase (I) has a parallel arrangement of molecules typical of the odd alkanes presented here. This odd-even variation will be expected to have profound consequences in the mixing behaviour of odd and even alkanes on the surface.

\section{Acknowledgement}

The authors thank UK EPSRC (TA), The Spanish DGICYT, The Leverhulme Trust (SMC) and The Yamada Science Foun- dation (AI) for financial support and the staff and scientists at the ILL for beam time and technical assistance.

\section{References}

1 T. Arnold, R. K. Thomas, M. A. Castro, S. M. Clarke, L. Messe and A. Inaba, Phys. Chem. Chem. Phys., 2002, 4, 345-351.

2 K. Morishige, Y. Tajima, S. Kittaka, S. M. Clarke and R. K. Thomas, Mol. Phys. 1991, 72, 395-411.

3 S. M. Clarke, Structure and Properties of Adsorbed Layers by $X$ ray and Neutron Scattering, D. Phil., University of Oxford, 1986.

4 S. M. Clarke and R. K. Thomas, Mol. Phys., 1991, 72, 413-423.

5 ILL "Neutron Research Facilities at the ILL High Flux Reactor," Institut Laue-Langevin, 1996.

6 J. K. Kjems, L. Passell, H. Taub, J. G. Dash and A. D. Novaco, Phys. Rev. B, 1976, B13, 1446.

7 B. E. Warren, Phys. Rev., 1941, 59, 693.

8 M. A. Castro, S. M. Clarke, A. Inaba, T. Arnold and R. K. Thomas, Phys. Chem. Chem. Phys., 1999, 1, 5203-5207.

9 A. J. Groszek, Proc. R.. Soc., London, Ser. A, 1970, A314, 473.

10 S. M. Clarke, A. Inaba, T. Arnold and R. K. Thomas, J. Therm. Ana/. Calorim., 1999, 57, 641-651.

11 M. A. Castro, S. M. Clarke, A. Inaba, T. Arnold and R. K. Thomas, J. Phys. Chem. B, 1998, B102, $10528-10534$.

12 H. Mathisen, N. Norman and B. F. Pedersen, Acta. Chim. Scand., 1967, 21, 127-134.

13 R. A. Bucknall, S. M. Clarke, R. A. Shapton and R. K. Thomas, Mol. Phys., 1989, 67, 439.

14 A. I. Kitaigorodskii, Organic chemical crystallography, Consulktants Bureau, New York, 1961.

15 J. P. Rabe and S. Buchholz, Science, 1991, 424, 253. 